\title{
Psicosis en el embarazo y posparto, manejo actual. Revision bibliografica
}

\author{
Psychosis in pregnancy and postpartum, current handling. \\ Bibliographicalrevsion
}

Diana Estefania Salazar Flores. ${ }^{1}$, Juan Carlos Salazar Flores. ${ }^{2}$, Paul Roberto Salazar Robalino. ${ }^{3} \&$ Johanna Estefanía Suquilanda Toapanta. ${ }^{4}$

DOI: $\underline{\text { https://doi.org/10.33262/anatomiadigital.v4i3.1792 }}$

\begin{abstract}
.
Resumen.

Introduction. Mental illness is a Introducción. La enfermedad mental en challenge for medicine, since the doctor un desafío para la medicina, ya que el must trust what the patient tells him since there are no visible signs to reach a médico debe confiar en lo que le dice el paciente ya que no hay signos visibles diagnosis. The best therapeutic option is necessary to treat the psychiatric para llegar a un diagnóstico. Es necesario la mejor opción terapéutica para tratar la pathology around pregnancy without harming neither its development nor the woman's own health. In the same way, it is to be able to have management guidelines for women who develop psychosis in the puerperal period in order to maintain an adequate quality of life for patología psiquiátrica en torno a la gestación sin con ello perjudicar ni el desarrollo de la misma ni la salud propia de la mujer. De igual manera es poder tener directrices de manejo para las mujeres que desarrollan psicosis en el periodo puerperal en aras de mantener

1 Medico General, Universidad Técnica de Ambato, Medico de consulta particular, Correo: diansalazar96@hotmail.com, (iD https://orcid.org/0000-0002-1206-8227

2 Medico Posgradista de Medicina Interna, Universidad UESS Samborondón, Clínica Guayaquil, Correo: juank8sf@yahoo.es, https://orcid.org/0000-0001-8079-0636

${ }^{3}$ Medico General, Universidad Técnica de Ambato, Hospital IESS Puyo, Correo: paulsr0988@gmail.com (iD) https://orcid.org/0000-0002-5387-4057

4 Estudiante de Medicina, Universidad Uniandes, Hospital General Ambato IESS, Correo: chocoleyt03_09@hotmail.com, (D) https://orcid.org/0000-0003-1470-6277
\end{abstract}


both the mother and the newborn and their families; An adequate and even more personalized management protocol has not been established for pregnant and postpartum patients who decline in their mental state, leading to the establishment of a psychiatric pathology that, without adequate follow-up, triggers a serious health problem. The main objective is to promptly recognize the different psychosis disorders during pregnancy and postpartum to be able to give their appropriate treatment, since in our environment it is very lightly taken by non-psychiatric professionals and thus initiate an awareness of general practitioners of the current problem of non-organic, but mental field of the patients. The methodology of this research work is carried out around bibliographic reviews in the medical literature on the psychiatric repercussion that occurs in certain pregnant women as well as in mothers in the immediate, mediate and late puerperal period. Results. Post-traumatic stress syndrome shows a prevalence of $1 \%$, appears between 24 and 48 hours after a difficult delivery. Postpartum depression there is a prevalence of $50-70 \%$ or even $80 \%$ of births on the third day postpartum, but it does not usually exceed a week. Postpartum depression in $10-15 \%$ of women in the first 2-3 months after delivery. Postpartum psychosis occurs in about 1-2 per 1,000 births, although other authors determine 3 per 1,000.

Keywords: Pathology. Therapy. Gestation. Woman. Puerperal. Neonate una adecuada calidad de vida tanto para la madre como para el neonato y sus familias; no se ha establecido un protocolo adecuado de manejo y más aún personalizado para aquellas pacientes gestantes y puérperas que declinan en su estado mental conllevando a establecer una patología psiquiátrica que sin un seguimiento adecuado desencadena en un grave problema de salud. El objetivo principal es reconocer con prontitud los diferentes trastornos de psicosis durante el embarazo y posparto poder dar su tratamiento adecuado, ya que en nuestro medio es muy tomado a la ligera por los profesionales no psiquiátricos e iniciar así una concientización a los médicos generales de la problemática actual de ámbito no orgánico, sino mental de los pacientes. La metodología del presente trabajo investigativo se realiza en torno a revisiones bibliográfica en la literatura médica sobre la repercusión psiquiátrica que se presenta en determinados gestantes así como en madres en periodo puerperal inmediato, mediato y tardío. Resultados. Síndrome de estrés postraumático muestra una prevalencia de $1 \%$, aparece entre las 24 y 48 horas después de un parto dificultoso. Depresión del puerperio hay una prevalencia de 50-70 \% o incluso $80 \%$ de los nacimientos al tercer día del posparto, pero no suele superar la semana. Depresión posparto entre 10-15\% de las mujeres en los 2- 3 primeros meses luego del parto. Psicosis posparto se presenta en alrededor de 1-2 por cada 1000 nacimientos, aunque otros autores determinan 3 por cada 1000 .

Palabras claves: Patología. Terapéutica. Gestación. Mujer. Puerperal. Neonato 


\section{Introducción}

El presente trabajo investigativo como revisión bibliográfica se ha instaurado con el fin de dar luces a un problema que, siendo muy frecuente en nuestro medio, no se ha establecido un protocolo adecuado de manejo y más aún personalizado para aquellas pacientes gestantes y puérperas que declinan en su estado mental conllevando a establecer una patología psiquiátrica que sin un seguimiento adecuado desencadena en un grave problema de salud.

Por ende, la problemática a resolver es la determinación fisiopatológica que desencadena en una gestante la aparición de eventos psicóticos que repercuten en un inadecuado control prenatal e inclusive con la pérdida del producto.

Desde los albores de la historia de la psiquiatría, se ha sabido que el nacimiento de un hijo puede acarrear complicaciones psiquiátricas. Es así como, desde los principios de la edad clásica, en Lorete (2018) se indica que Hipócrates en la antigüedad ya describió las enfermedades mentales graves que aparecían después del parto, a las que denominó "locura de las parturientas".

Desde los finales del siglo XVI y en el siglo XVII, los expertos alemanes en Rechtsmedizin (medicina legal) produjeron múltiples estudios sobre el escabroso tema del neonaticidio, tan prevalente en Europa durante varios siglos. En el año 1757 un médico inglés describió en los Proceedings of the Royal Society, obsesiones de infanticidio. Esta fue la primera mención de un grupo de trastornos de ansiedad que ahora incluyen la tocofobia, el pánico puerperal, fobia hacia el bebé, miedo patológico de muerte súbita del niño, dismorfofobia pre y postparto, el trastorno por estrés postraumático y el trastorno querulante (litigioso) que pueden ser consecuencia de un parto traumático. (Liji, 2019)

Pero es a partir del siglo XIX cuando las manifestaciones psíquicas unidas al puerperio se individualizan y en 1895, MARCE expone claramente el problema de la especificidad etiológica, clínica y evolutiva de las psicosis puerperales. (Moure, 1997)

Es imprtante saber el concepto de buena salud perinatal para prevenir alteraciones entre las buenas relaciones sociales, psicológicas, ambientales y biológicas. Es imprtante agendar la cita postparto antes de las seis semanas en mujeres que reflejen síntomas depresivos o psicóticos en el postparto inmediato para detección temprana de quienes cursan con una depresión de base, o presenten historia de depresión o psicosis postparto previa. Una hospitalización temprana es el manejo principal del paciente para asegurar su bienestar y el del neonato, además de tratamiento farmacológico con medicamentos antipsicóticos y terapia de cualquier trastorno subyacente. Aunque los episodios de psicosis durante el embarazo son raros, las mujeres con historial de esta condición tienen un mayor riesgo de reincidencia.

Un estudio encontró que el embarazo parece empeorar la salud mental de la madre con esquizofrenia. Algunas mujeres presentan negación psicótica del embarazo, síntoma que 
de no ser abordado puede representar un factor de riesgo. En el postparto, estas mujeres pueden ser especialmente susceptibles a exacerbaciones agudas de la esquizofrenia. (Arrate, Molina, \& Linares , 2016)

Por otro lado, un metaanálisis que contó con datos de 12 estudios que incluyeron a 700 sujetos con esquizofrenia y 835 sujetos en el grupo control, encontró asociaciones significativas entre el trastorno y la ruptura prematura de la membrana, edad gestacional menor a 37 semanas y uso de incubadora y resucitación. Otra investigación también encontró que tenían más riesgo de parto prematuro y, además, la esquizofrenia durante el embarazo se asociaba a bajo peso al nacer. (Bas, 2017)

En cuanto al tratamiento, la psicoeducación podría reducir el riesgo de complicaciones en el embarazo. Las terapias breves enfocadas también pueden ser útiles para algunas pacientes con dicha condición psicológica. Con respecto al tratamiento farmacológico, un estudio de dos casos de mujeres medicadas con clozapina para la esquizofrenia durante el embarazo no observó riesgos en el embarazo, parto, la salud de la madre ni del bebé.

Para Fonseca (2018) "La psicosis postparto suele presentarse luego de dos semanas del nacimiento, siendo delirios y alucinaciones junto con pensamiento desorganizado los síntomas pivotes que caracterizan el cuadro. Durante este trastorno también se pueden presentar otras alteraciones del estado de ánimo entre los que se encuentran: ansiedad, irritabilidad, agitación psicomotora y dificultad para conciliar el sueño no asociado al recién nacido. Existen otros trastornos psiquiátricos que con frecuencia se relaciona a la psicosis postparto".

La psicosis posparto puede presentarse como un estado maníaco, depresivo o mixto entre los pacientes con trastorno bipolar. Las mujeres con trastorno bipolar tienen un alto riesgo de recidiva en el embarazo y después del parto, que puede presentarse como psicosis posparto. Las mujeres que dejan los medicamentos estabilizadores del ánimo antes o durante el embarazo pueden tener un mayor riesgo de psicosis posparto. Para las mujeres sin historia previa, un episodio psicótico después del parto puede ser la primera manifestación de un trastorno bipolar. (FonsecaVillanea, 2018)

La psicosis postparto requiere un reconocimiento temprano, con un manejo pluridisciplinario. Se debe tener claro que la psicosis puerperal es una urgencia psiquiátrica, pues compromete el pronóstico de madre-hijo, por el riesgo de agresión que presenta el trastorno.

Actualmente, se está desarrollando el concepto de psiquiatría perinatal que tiene como objetivo ayudar a las madres a prevenir lo más precozmente posible las alteraciones psiquiátricas posteriores al parto y garantizar un buen desarrollo mental del neonato. (FonsecaVillanea, 2018)

En la actualidad se sabe que alrededor del $50 \%$ de los episodios de depresión posparto comienza en realidad en el embarazo por lo cual; En 2013, la versión más reciente de la clasificación de enfermedades mentales de la Asociación Americana de Psiquiatría 
(DSM-5), recogió estos avances y, en los criterios para Depresión Mayor, sustituyó el antiguo especificador "de comienzo en el posparto" por el especificador "con inicio en el periparto". Esta denominación se puede utilizar para especificar que el episodio depresivo mayor se presentó durante el embarazo o en las cuatro semanas posteriores al parto. (Jadresic, 2017)

El desafío es enorme, pero podemos ser optimistas. La ignorancia, el pesimismo ya no se justifican. Estamos en una posición mucho más sólida para comprender lo que están padeciendo estas mujeres con trastornos y para intervenir eficazmente. Actualmente se puede hacer mucho para restablecer su salud mental plena. Todo esfuerzo que hagamos para mejorar el bienestar y el ánimo de las madres es una contribución a la vida de la familia y la salud de la generación siguiente. (Brockington I. , 2017)

\section{Metodología}

El presente trabajo investigativo se realiza en torno a revisiones bibliográfica en la literatura médica sobre la repercusión psiquiátrica que se presenta en determinados gestantes, así como en madres en periodo puerperal inmediato, mediato y tardío.

Tras la revisión bibliográfica, se analizaron varios artículos, entre los años 2016 hasta la actualidad, de los cuales nos enfocamos alrededor de 15 artículos por su pertinencia, los cuales muestran solilitud con el presente estudio. Se utilizaron para ello las bases de datos enfermeras Library, Medigrafic, Psiquiatria, Revista medica clinica Condes, psiquiatriafacmed, Revista Médica Sinergia, Pubmed, Dialnet, Cochrane y Scielo. y el metabuscador Google Académico.

Se realizo una revisión de las referencias en los artículos seleccionados.

Se hizo una búsqueda específica en referecia a cada base de datos, empleando la combinación de operadores booleanos de los siguientes terminos controlados: "Embarazo" (Pregnancy), "Posparto" (Postpartum period), "Trastornos psicóticos" (Psychotic disorders).

Se utilizaron criterios de inclusion como son: estudios que analizaran la etiología de la psicosis puerperal y ensayos clínicos controlados aleatorios que investigaran los efectos de las opciones de tratamiento donde esta incluida la atencion psicosocial estándar, fármacos antipsicóticos, terapia cognitivo conductual y manejo actual integral en mujeres gestantes y en periodo postparto con diagnósticos de trastornos psicóticos. Sin limitacion idiomatica ni temporal.

\section{Resultados y discusión}

Se seleccionaron 7 artículos científicos para la presente revisión bibliográfica. En cuanto al diseño de los artículos hubo 5 artículos de revisión, dos de los cuales constan en la base de datos COCHRANE, 2 de MEDIGRAFIC, PERINATOLOGIA REPRODUCCION HUMANA, 1 revisión de la ASOCIACION GALLEGA DE PSIQUIATRIA, un caso clínico en MEDISAN, 1 revision tomada del portal BIBLIOPSIQUIS. 


\section{Factores predisponentes}

Los trastornos del estado de ánimo del puerperio: tristeza postparto, depresión postparto y psicosis postparto tienen una alta prevalencia. Éstos afectan directamente la salud física y emocional de la madre, al igual que la del recién nacido y la pareja, produciendo inestabilidad en el entorno familiar y social de quien la padece. (Medina-Serdán, 2013)

De acuerdo a Arrete, Molina, Linares(2016), existen factores predisopinentes que se pueden menciohnar como:

Síndrome de estrés postraumático: Muestra una prevalencia de $1 \%$ y aparece normalmente entre las 24 y 48 horas después de un parto dificultoso. Se presenta con ansiedad asociada con pesadillas e imágenes recurrentes de la experiencia vivida. Es un cuadro pasajero; escasas veces se cronifica. No necesita tratamiento específico.

Depresión del puerperio: Estado de ánimo melancólico, larvado, que puede pasar inadvertido. Se notifica una prevalencia de 50-70\% o incluso $80 \%$ de los nacimientos. Comienza alrededor del tercer día del posparto, pero no suele superar la semana. Si se intensifican los síntomas o duran más de un mes, se debe valorar el riesgo de cronificación.

Depresión posparto: Afecta entre 10-15 \% de las mujeres en los 2- 3 primeros meses luego del parto; resulta la complicación médica más habitual de este período. El cuadro sintomático es el típico de una depresión: sentimiento de culpa, trastornos del sueño, cambios de humor con gran tendencia a la tristeza y ausencia de síntomas psicóticos. Mejor pronóstico que las depresiones de otra causa, pero en casos graves requiere hospitalización. Entre otros factores de riesgo se citan: edad materna (menor de 20 años y mayor de 30), problemas psiquiátricos o psicológicos durante el embarazo, problemas psiquiátricos previos al embarazo, tensiones en la pareja durante el embarazo, acontecimientos desfavorables que generen ansiedad, vividos durante el puerperio.

Psicosis posparto: se puede confundir con una depresión; su rasgo diferencial es la presencia de síntomas típicamente psicóticos, como delirios o alucinaciones. La duración es variable y en algunos casos se requiere la hospitalización. Se presenta en alrededor de 1-2 por cada 1000 nacimientos, aunque otros autores elevan esta cifra a 3 por cada 1 000. El cuadro clínico consiste en depresión e ideas delirantes que aparecen después del parto, así como otras características dadas por el rápido cambio de los síntomas, la labilidad de humor y, con frecuencia, los signos de confusión.

Los factores que aumentan el riesgo de psicosis posnatal incluyen madres primíparas solteras, mujeres mayores, o con antecedentes psiquiátricos previos y antecedentes familiares de psicosis afectiva, depresión prenatal y disfunción tiroidea autoinmune. (Adib, 2013) 
Intervenciones preventivas para la psicosis posnatal

Ante la problemática establecida y estudiada en Latinoamérica se puede inferir que la misma es un problema en común denominador que hace referencia a la idiosincrasia de cada pueblo y la practica sanitaria. Desde luego que con ello se evidencia la repercusión en el núcleo familiar a través de no solamente el alto gasto para tratamiento medicamentoso y psicológico sino por el mismo hecho hay una repercusión directa a nivel social ante ausentismo laborar, bajo rendimiento laboral entre otros factores.

\section{El periodo gestacional.}

Durante el embarazo, y de forma fisiológica, aparecen en la mujer modificaciones afectivas debido a los cambios hormonales y a las expectativas vitales y las fantasías ante el nacimiento de un hijo. (Linares Despaigne, 2016)

Estos cambios son más aparentes en la primera gestación, aunque no son exclusivos de esta y pueden reproducirse en cierta medida en cada nueva maternidad. (Linares Despaigne, 2016)

Las cifras de riesgo de presentar un trastorno psiquiátrico durante el embarazo es, por año, de 7,1 cada 100000 embarazadas. (Linares Despaigne, 2016)

\section{El periodo puerperal.}

La psicosis posnatal es una enfermedad global potencialmente mortal que afecta de una a dos de cada 1000 nuevas madres. Tiene una aparición abrupta dentro del mes posterior al parto. Las nuevas madres afectadas desarrollan rápidamente psicosis manifiesta, deterioro cognitivo y comportamientos desorganizados.

Se han descrito alrededor de 5000 casos de psicosis puerperal en la literatura mundial, aproximadamente $250,5 \%$ han sido de etiología infecciosa. Una proporción de 7 a $9 \%$ se mantuvo estable hasta el descubrimiento de las sulfonamidas en 1936, desde entonces dicha cifra ha disminuido hasta que estas entidades casi se han olvidado. (Brockington I. , 2008)

El riesgo de una recurrencia posnatal futura es del $25 \%$ al $57 \%$. Las intervenciones preventivas para la psicosis posnatal tienen como objetivo identificar a las pacientes con factores de riesgo, lograr el reconocimiento temprano de la psicosis inminente mediante el cribado, e incluyen el uso de tratamiento farmacológico preventivo. Los estabilizadores del estado de ánimo, los fármacos antipsicóticos y la terapia hormonal pueden tener efectos beneficiosos en la prevención de los episodios psicóticos posnatales en las pacientes con riesgo. (Adib, 2013)

La mayoría de las psicosis del posparto son psicosis afectivas, revestidas en el $20 \%$ de los casos aproximadamente, de unas conductas de desorganización de la conciencia y de fluctuaciones del humor agudas y rápidas que debutan bruscamente en las primeras semanas del posparto. (Moure, 1997) 


\section{Manejo psicológico y psiquiátrico.}

La morbilidad causada por la depresión puerperal es enorme. Varias intervenciones psicológicas y psicosociales parecieron ser efectivas para tratar este trastorno, aunque ninguna mostró un beneficio claro para prevenir el desarrollo de la depresión puerperal. Sin embargo, hasta ahora no se ha evaluado la efectividad de la hipnosis en relación con este trastorno. (Sado, 2012)

La litioterapia no debe ser iniciada según Stewart antes del parto, por los posibles efectos secundarios en el recién nacido (hipotonía, cianosis, letargia...). Este tratamiento con litio necesita una vigilancia intensa de las litemias, por el riesgo de sobredosis unida a las modificaciones electrolíticas del posparto. (Moure, 1997)

Brew y Seidemberg recomiendan no utilizar la terapia electroconvulsiva hasta después de las 4 semanas del parto, para evitar complicaciones como la movilización de un émbolo a partir de una tromboflebitis no diagnosticada (Moure, 1997)

Este tipo de trastornos requiere una estrategia terapéutica para controlar de un modo integral varios aspectos, como los síntomas maternos, por un lado, mediante el uso de técnicas psicológicas, apoyadas, según lo requiera el caso, con medicación psicótropa, como los neurolépticos, junto con benzodiazepinas o antidepresivos si existe un mayor componente ansioso o depresivo. (Linares Despaigne, 2016)

\section{Pronostico}

El pronóstico es generalmente favorable con una curación en el 70 a $80 \%$ de los casos. Los estudios de larga duración indican que de un 10 a un 15\% de las psicosis puerperales evolucionan hacia una esquizofrenia crónica. (Moure, 1997)

\section{Conclusiones}

- Los trastornos psiquiátricos en el embarazo son tan frecuentes como 1-2 de cada 1000 nuevas madres y distribuido a nivel mundial

- Existe un alto riesgo de recurrencia en la presentación de psicosis en embarazos posteriores de hasta $57 \%$

- Hasta un $20 \%$ de las psicosis en el embarazo tienen como etiología conductas de desorganización de conciencia

- Los diagnósticos diferenciales hacia la psicosis posparto se los tiene con tristeza y depresión puerperales siendo el primero motivo de seguimiento hospitalario y motivo de tratamiento pscico- farmacológico más complejo

- Se ha asociado hasta un 5\% de etiología infecciosa durante el parto para el aparecimiento de trastorno psiquiátrico.

\section{Referencias bibliográficas}

Moure, R. (1997). LAS PSICOSIS PUERPERALES . ASOCIACION GALLEGA DE PSIQUIATRIA . 
Medina-Serdán, E. (2013). Diferencias entre la depresión postparto, la psicosis postparto y la tristeza postparto. PERINATOLOGÍA Y REPRODUCCIÓN HUMANA, 185-193.

Adib, E. e. (2013). Intervenciones preventivas para la psicosis posnatal. Cochrane Library

Sado, M. e. (2012). Hipnosis durante el embarazo, el parto y el periodo posnatal para prevenir la depresión puerperal. Cochrane Library.

Brockington, I. (2008). La importancia actual de las psicosis organicas duarente el embarazo, parto y puerperio . PERINATOLOGIA, REPRODUCCION HUMANA, 36-46.

Jadresic, E. (2017). DEPRESIÓN POSPARTO EN EL CONTEXTO DEL HOSPITAL GENERAL. Revista médica Clínica Las Condes, 28(6), 874-880. doi:10.1016/j.rmclc.2017.10.007

Bas, I. (23 de 03 de 2017). Psicosis Puerperales (2017). Obtenido de https://rdu.unc.edu.ar/bitstream/handle/11086/265/bas_isaia_sr.pdf?sequence=1

Brockington, I. (12 de 02 de 2017). La importancia actual de la psicosis organicas durante el embarazo, parto, puerperio (2017). Obtenido de Revista Perinatal de Reproducion

Humana: https://www.inper.mx/descargas/pdf/ImportanciaActualdelaspsicosisOrgasmicas Duranteel.pdf

Liji, T. (19 de 02 de 2019). Psicosis postparto/postnatal (2019). Obtenido de News Medical Life Sciences: https://www.news-medical.net/health/PostpartumPostnatal-Psychosis-(Spanish).aspx

Lorete, G. (01 de 05 de 2018). CONOCER LA DEPRESIÓN Y LA PSICOSIS EN EL EMBARAZO Y PUERPERIO. Obtenido de Congreso Internacional de Salud Mental: https://psiquiatria.com/congresos/pdf/3-3-2017-4-pon2.pdf

Hernández, R. (2020). Psicosis postparto: a propósito de un caso. Psiquiatria, 24, $1137-$ 3148. doi:http://psiqu.com/1-10496

FonsecaVillanea, C. (2018). PSICOSIS POSTPARTO. Revista Médica Sinergia, 3(8), 712. https://revistamedicasinergia.com/index.php/rms/article/view/134/338

Arrate, M., Molina, V., \& Linares, M. (2016). Tratamiento ambulatorio en una paciente con psicosis puerperal. MEDISAN, 20(11), 2390. Recuperado de http://scielo.sld.cu/pdf/san/v20n11/san102011.pdf

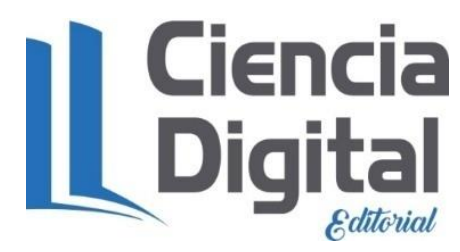




\section{PARA CITAR EL ARTÍCULO INDEXADO.}

Salazar Flores, D. E., Salazar Flores, J. C., Salazar Robalino, P. R., \& Suquilanda Toapanta, J. E. (2021). Psicosis en el embarazo y posparto, manejo actual. Revision bibliografica. Anatomía Digital https://doi.org/10.33262/anatomiadigital.v4i3.1792

\section{Ciencia \\ LDigital}

El artículo que se publica es de exclusiva responsabilidad de los autores y no necesariamente reflejan el pensamiento de la Revista Anatomía Digital.

El artículo queda en propiedad de la revista y, por tanto, su publicación parcial y/o total en otro medio tiene que ser autorizado por el director de la Revista Anatomía Digital.
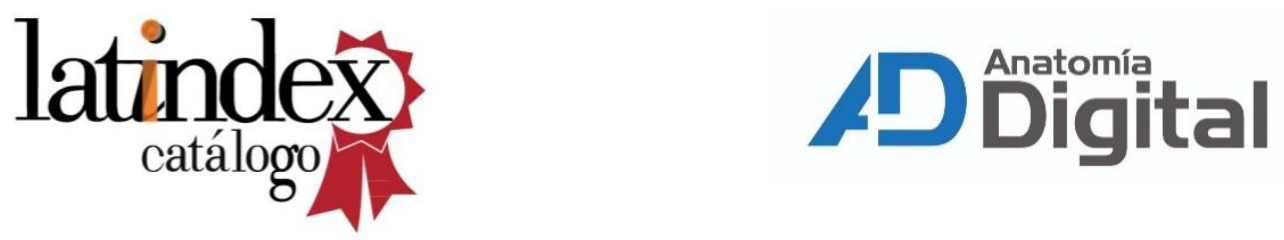